\title{
Pancreatic pleural effusion masquerading as right sided tubercular pleural effusion
}

\author{
Gopal Chawla ${ }^{1}$, Ram Niwas ${ }^{1}$, Nishant Kumar Chauhan ${ }^{1}$, Naveen Dutt ${ }^{1}$, Taruna Yadav ${ }^{2}$, Priyank Jain ${ }^{1}$ \\ ${ }^{1}$ Department of Pulmonary Medicine, All India Institute of Medical Sciences, Jodhpur; ${ }^{2}$ Department of Radiology, All \\ India Institute of Medical Sciences, Jodhpur, India
}

\begin{abstract}
Pleural effusion is easily diagnosed often managed optimally with standard protocols. It at times, is a diagnostic dilemma as it comes with big list of differential diagnosis. Pleural effusion due to pancreaticopleural fistula (PPF) is a rare and on right side is even rarer. Detailed history along with high index of suspicion in required to diagnose PPF, which is confirmed by increased level of pleural fluid amylase and lipase along with magnetic resonance cholangiopancreatography (MRCP) or endoscopic retrograde cholangiopancreatography (ERCP) demonstrating fistula tract. Here we report the case of a young patient who presented with respiratory distress and was wrongly diagnosed as right sided tubercular effusion which later turned out to be pancreatic effusion. Management in our case was multi-disciplinary involving pulmonologist, gastroenterologist, radiologist and thoracic surgeon.
\end{abstract}

Correspondence: Dr. Ram Niwas, Department of Pulmonary Medicine, All India Institute of Medical Sciences, Jodhpur, India. Mobile: +91.9999493179 .

E-mail: rniwasaiims@gmail.com.

Key words: Pancreatic-pleural fistula; VATS; ERCP

Conflict of interest: The authors declare no conflict of interest.

Contributions: GC, RN, NKC, study concept and design, definition of intellectual contents; GC, RN, PJ, literature search; TY, PJ, data acquisition; TY, data and statistical analysis; GC, RN, NKC, ND, PJ, manuscript drafting, editing and review. All authors read and approved the final version to be published.

Received for publication: 3 July 2019

Accepted for publication: 25 August 2019.

${ }^{\circ}$ Copyright: the Author(s), 2019

Licensee PAGEPress, Italy

Monaldi Archives for Chest Disease 2019; 89:1125

doi: 10.4081/monaldi.2019.1125

This article is distributed under the terms of the Creative Commons Attribution Noncommercial License (by-nc 4.0) which permits any noncommercial use, distribution, and reproduction in any medium, provided the original author(s) and source are credited.

\section{Case Report}

A 35-year-old male reformed-smoker was referred to our tertiary centre with a history of right sided chest pain, dry cough, and breathlessness for 2 months. Chest X-ray showed moderate right sided pleural effusion. Pleural fluid analysis showed lymphocytic exudative pleural effusion with high adenosine deaminase (ADA) of $47 \mathrm{IU} / 1$ (cut off for tubercular pleural effusion $\geq 40$ ). The patient also resulted positive at the Mantoux test; for this he had undergone therapeutic pleural tapping and still he was in respiratory distress. He was started on anti-tubercular treatment, to which he was not responding even after a month. Repeat chest radiograph revealed right sided massive pleural effusion; ultrasound chest showed massive pleural effusion with multiple loculations; while other routine investigations were within normal limits. While ruling out other differential diagnosis for pleural effusions, the patient reported bulky foul-smelling stools, which were difficult to flush. Serum amylase and lipase tests were performed, which resulted 248 IU/1 (normal value 23-85) and 89 IU/1 (0-160), respectively. Ultrasonography of abdomen showed shrunken irregular and multiple foci of calcifications which were consistent with chronic calcific pancreatitis. Repeat diagnostic thoracocentesis revealed haemorrhagic, exudative effusion with high ADA 51 U/1 and high levels of pancreatic enzymes: amylase, 12257 IU/1 and lipase, $>75 \mathrm{k} \mathrm{IU} / 1$. Elevated pancreatic enzymes in pleural fluid pointed towards the presence of pancreatic effusion. Due to rapid accumulation and recurrence of effusion, pancreaticopleural fistula was suspected. Though it is common on left but here effusion was right sided. Hence, the patient was planned for contrast enhanced tomogram (CECT) thorax and abdomen, which showed atrophic pancreas with multiple calcifications with dilated pancreatic duct suggestive of chronic calcific pancreatitis along with gross right sided pleural effusion with diffuse thickening of parietal pleura. A small hypodense collection measuring $4 \times 2.5 \mathrm{~cm}$ is seen along superior surface of tail of pancreas which extend into right pleural cavity across diaphragm suggestive of pancreaticright pleural fistula (pancreatic-right pleural fistula was confirmed on MRI abdomen) through diaphragmatic hiatus (Figure 1).

For management various options were weighed, and the patient was initially managed conservatively with subcutaneous octreotide $100 \mathrm{mcg}$ three times a day. As the patient was in respiratory distress due to rapid refilling, tube thoracostomy was done. Haemorrhagic fluid was continuously drained through the chest tube and the daily output was ranging from 200 to $300 \mathrm{ml}$ for 2 weeks with pleural fluid amylase level of 308 IU/1. Due to persistence of drainage, an endoscopic retrograde cholangiopancreatography (ERCP) was performed, which showed dilatation of main pancreatic duct and side branches. On same setting a 7 Fr $12 \mathrm{~cm}$ straight pancreatic stent was inserted. After stent placement, fistu- 
la output from the chest tube reduced markedly to $10 \mathrm{ml}$ per day over 20 days. Unfortunately, the chest radiology showed no significant changes, as there were multiple fibrotic bands. Therefore, the patient underwent a video assisted thoracoscopic surgery (VATS) guided adhesiolysis and decortication, after which lung expanded partially and responded further to negative suction (Thopaz +; Medela). Later chest tube was removed when lung fully expanded and there was no drainage. The patient is presently under followup and is doing fine even after a year (Figure 2).

\section{Discussion}

Pleural effusion is easily diagnosed, more often than not optimally managed with standard protocols but at times, it is a diagnostic dilemma as it comes with big list of differential diagnosis. Pleural effusion due to pancreaticopleural fistula (PPF) is rare as it accounts for less than $1 \%$ of cases and it needs to be distinguished from self-limiting reactionary pleural effusions that can manifest in $3-7 \%$ of patients with pancreatitis [1].

Pathogenetic mechanism development of PPF is often a result of leak from an incompletely formed or ruptured pseudocyst or direct pancreatic duct leak. The fistulous tract passes either through the aortic or oesophageal diaphragmatic orifice or directly transdiaphragmatically. If the pancreatic duct disruption occurs anteriorly and is not walled off PPF developed will result in ascites. If this happens posteriorly, pancreatic secretion leak into retroperitoneum and may traverse through aortic or oesophageal hiatus into mediastinum and form a pleural fistula or at times present as mediastinal pseudocyst which in turn ruptures into the pleural cavity and forms a pleural fistula [2].

Diagnosis of pancreatic-pleural fistula is often delayed unless there is suspicion especially in alcoholic patients or known cases of chronic pancreatitis along with pleural effusion. Duration of delay in diagnosis usually ranges from 12 to 49 days [3]. In above described case where patient presented with massive right sided effusion added to the difficulty in diagnosis because pancreaticpleural fistula in maximum number of cases is described with left sided effusion. Diagnosis in our case was even more difficult due to the predominance of pleuro-pulmonary symptoms more than abdominal symptoms like abdominal pain and vomiting. Adding to the difficulty was pleural fluid analysis which had high ADA, which in endemic country like India is very specific for tuberculo-
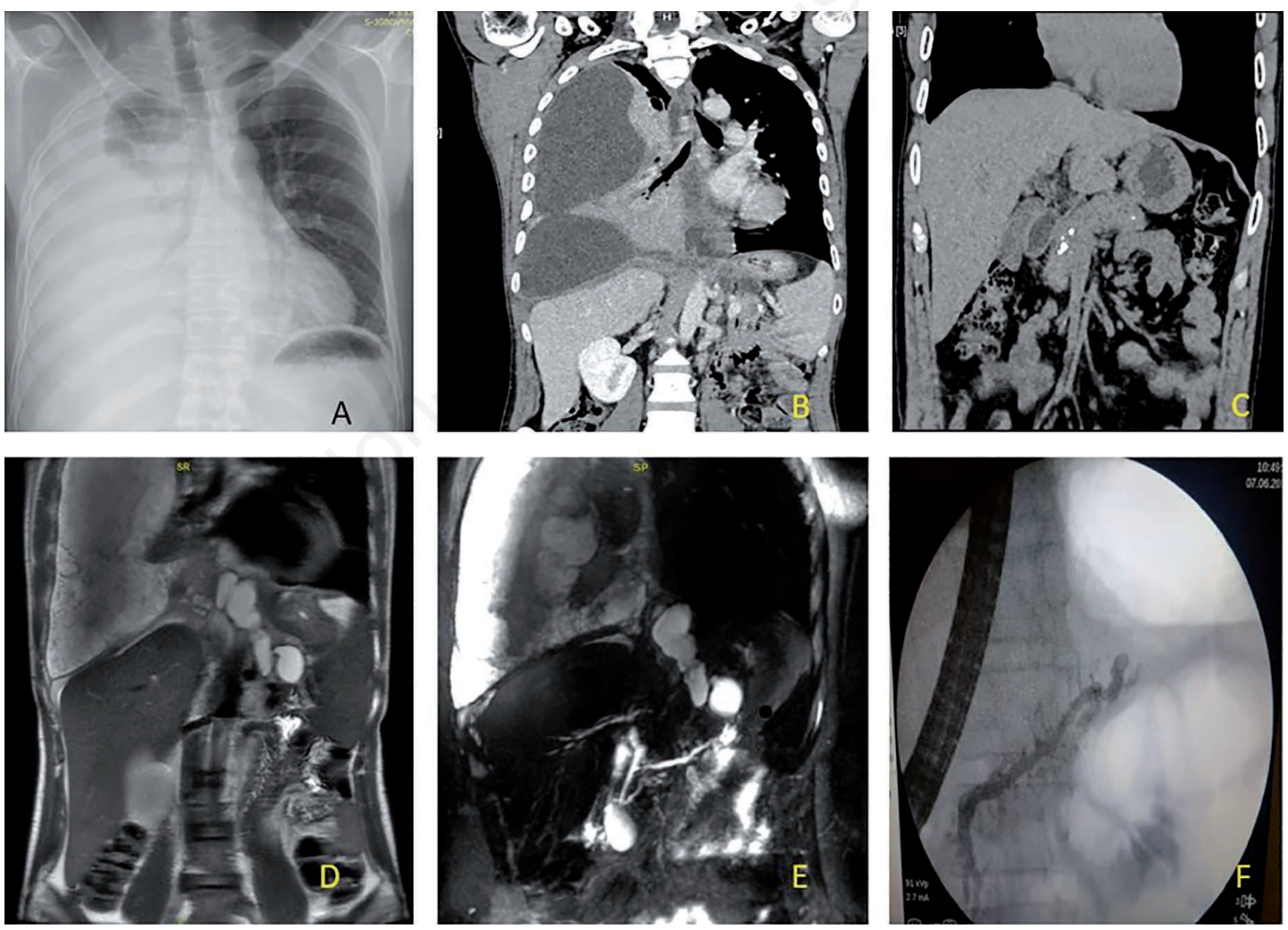

Figure 1. A) Chest X-ray showing right sided massive pleural effusion; B,C) CT chest showing collapse of right hiatus enlargement; chronic pancreatitis (D) and MRCP (E) show hyperintense tract in a close relation with distal main pancreatic duct, extending via hiatus into pleural cavity. F) ERCP image showing dilated main pancreatic duct. 
sis. ADA for tuberculosis has high sensitivity (81-100\%) and specificity (83-100\%). ADA seldom gets past cut off value in nontubercular pleural effusions. There are few exceptions like empyema, post $\mathrm{CABG}$ effusions and protein rich effusions [4]. Interpretation of pleural fluid ADA is tricky too as increases with pleural fluid protein. Pleural protein and LDH are indicators of the severity of pleural inflammation and there would be definitely more activated lymphocytes and ADA production in the presence of greater pleural inflammation [5].

Suspicion of PPF usually is on basis of the clinical picture and analysis of pleural fluid which reveals very high amylase level (normal $<150 \mathrm{IU} / \mathrm{L}$ ), lipase, and high albumin content $(>3 \mathrm{~g} / \mathrm{dL}$ ). The serum amylase is usually less elevated and is thought to be partly secondary to reabsorption of amylase from pleural surfaces. Other differentials for amylase-rich pleural effusion includes acute pancreatitis, cancer of lung, breast, rectum, female reproductive system, oesophageal perforation, lymphoma, leukaemia, hydronephrosis, liver cirrhosis, and pulmonary tuberculosis [6].

At times underlying pancreatic disease is frequently asymptomatic, [7] as in our case. Few patients may present with history of abdominal pain or steatorrhea, but the first clue to PPF more often than not is high pleural fluid amylase concentration that may require detailed radiologic imaging for confirming it. Abdominal sonogram and computed tomography usually show pseudocyst with evidence of chronic pancreatitis [8]. Non-invasive modality MRCP, has been shown to visualize the duct beyond the strictures, shows parenchymal atrophy along with ductal anatomy, pseudocyst, peripancreatic collection or PPF. Sensitivity of CT scans for PPF is low in comparison to MRCP and ERCP that have sensitivities around 80\%, MRCP seems to be the investigation of choice for diagnosing PPF [9].

First aim in management of PPF is to suppress pancreatic activity to help in resolution of the pleural effusion and thus closure of the fistula. Chest tube drainage and somatostatin or
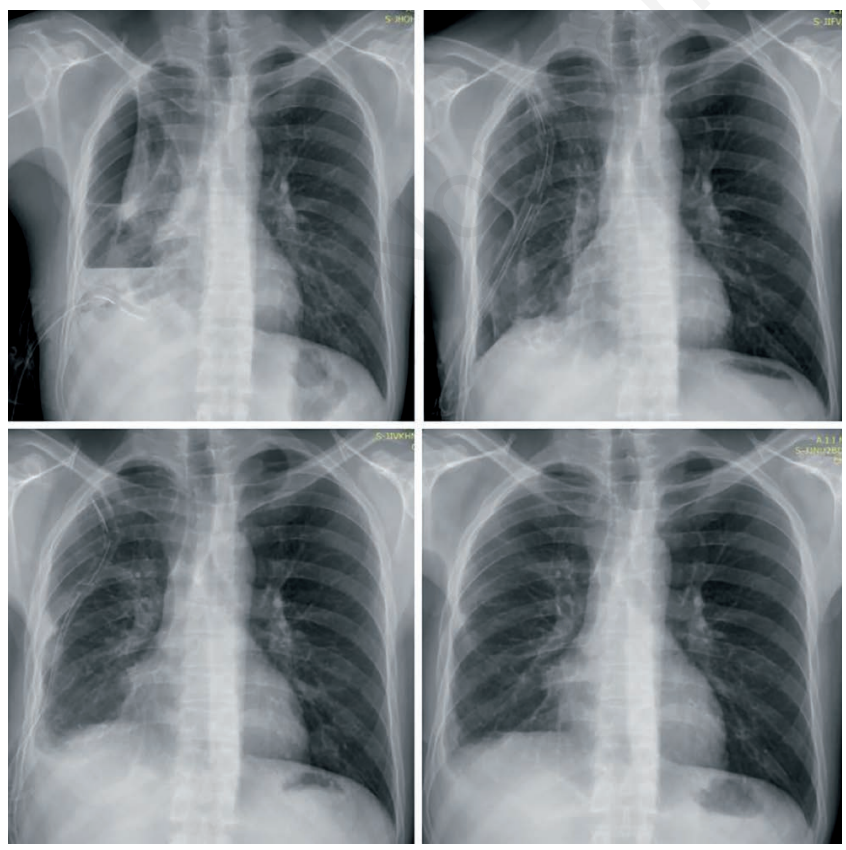

Figure 2. A) Chest radiograph post ICD placement showing multiple loculation; B) after VATS decortication; C) after negative pleural suction; D) after 2 months, completely expanded. octreotide infusion are successful in up to $40 \%$ of cases. It worked in our case too to some extent. Other measures like nil per oral and total parenteral nutrition have become obsolete as it involved many complications like malnutrition, deep vein thrombosis and intestinal mucosal atrophy from prolonged fasting [10]. So we needed the better option, ERCP and stent placement have given new turn to the concept of nonsurgical management in these patients. Potential benefits of ERCP include dilatation of stenosis of the main pancreatic duct, papillary sphincterotomy in cases of sphincter of Oddi dysfunction, and extraction of stones with or without extracorporeal lithotripsy and stent placement all of which could contribute to persistence of the fistula [11]. The main motive of stent placement other than decompressing the duct is to bridge the site of duct disruption if possible as most fistulae appear to arise from head or body of the pancreas and are thus amenable to bridging with a pancreatic stent. Most stents used are either $5 \mathrm{Fr}$ or $7 \mathrm{Fr}$ size we used $7 \mathrm{Fr}$ stent which resulted in decreasing the pleural effusion as fistulae from a pseudocyst which are no longer in communication with pancreatic ductal system heal spontaneously $[12,13]$.

Other option in such cases is surgery which is considered if there is persistence of the effusion, bacterial infection of the pseudocyst, and recurrence of the effusion after reintroduction of oral intake. A leak from the distal pancreatic duct or a pseudocyst in the tail of the pancreas can be managed by distal pancreatectomy. Pancreatic-jejunostomy is usually required for the more typical proximal duct rupture not responding to medical therapy. The aim of surgery is to achieve free internal drainage of the obstructed pancreatic duct and pseudocyst, allowing resolution of the pleural effusion and spontaneous closure of the fistula as they are associated with success rates of $80-90 \%$.

\section{Conclusions}

PPF is tricky to diagnose and at times difficult to treat. As it requires a high index of clinical suspicion with detailed history and examination. Early pleural fluid amylase testing can avoid delayed diagnosis. Management of PPF includes drainage of the effusion, octreotide and possibly ERCP with stenting of the pancreatic duct. Surgery is generally considered to be appropriate when medical measures fail or there is an associated pseudocyst. VATS can be considered in case of loculated pleural effusion with entrapped lung.

\section{References}

1. Materne R, Vranckx P, Pauls C, et al. Pancreaticopleural fistula: diagnosis with magnetic resonance pancreatography. Chest 2000;117:912-4.

2. Machado NO. Pancreaticopleural fistula: revisited. Diagn Ther Endosc 2012;2012:815476.

3. Uchiyama T, Suzuki T, Adachi A, et al. Pancreatic pleural effusion: case report and review of 113 cases in Japan. Am J Gastroenterol 1992;87:387-391.

4. Jiménez Castro D, Díaz Nuevo G, Pérez-Rodríguez E, Light RW. Diagnostic value of adenosine deaminase in nontuberculous lymphocytic pleural effusions. Eur Respir J 2003;21:220.

5. Tay TR, Tee A. Factors affecting pleural fluid adenosine deaminase level and the implication on the diagnosis of tuberculous pleural effusion: a retrospective cohort study. BMC Infect Dis 2013;13:546. 
6. Girbes ARJ, Postmus PE, Jansen W, et al. An alcoholic with pleural effusion. Eur Respir J 1990;3:934-6.

7. Rockey DC, Cello JP. Pancreaticopleural fistula: Report of 7 patients and review of the literature. Medicine 1990;69:332-44.

8. Verhaege W, Meysman M, Opdebeeck B, et al. Pancreaticopleural fistula: A case report. Eur J Radiol 1996;23: 118-20.

9. Ali T, Srinivasan N, Le V, Chimpiri AR, Tierney WM. Pancreaticopleural fistula. Pancreas 2009;38:e26-31.

10. Mihai C, Floria M, Vulpoi R, et al. Pancreatico-pleural fistula - from diagnosis to management. A case report. J Gastrointestin Liver Dis 2018;27:465-9.

11. Szary NM, Al-Kawas FH. Complications of endoscopic retrograde cholangio-pancreatography: how to avoid and manage them. Gastroenterol Hepatol (N Y) 2013;9:496-504.

12. Houlihan MD, Bowyer BA, Barclay RL. Resolution of pancreatico-pleural fistula with endoscopic ultrasound-guided therapy. Respir Med Case Rep 2013;9:30-3.

13. Shah HK, Shah SR, Maydeo AP, Pramesh CS. Pancreaticopleural fistula. Endoscopy 1998;30:314. 